
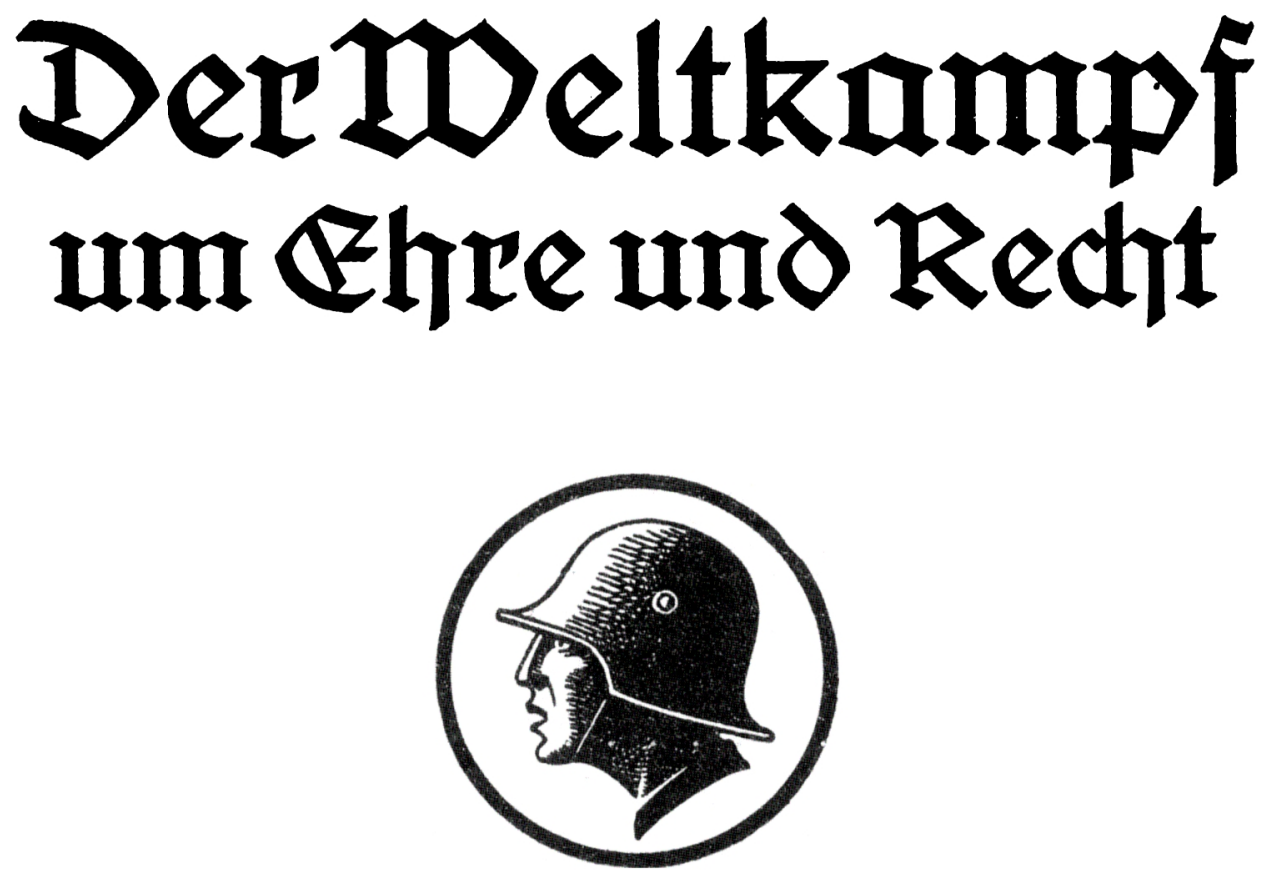

Die Exforjhung des Krieges in jeimer wahren Begebenheit, auf amtlicten $\mathfrak{u}$ rkunden und $7 k t e n$ beruhend *

Unter Betefligung Don 70 herborragenden Mitarbeitem herangyegeben von Ezzellenz Beneralleutnant $\mathfrak{M} \mathfrak{a} \mathfrak{z}$ d $\mathfrak{w} \mathfrak{a} \mathbf{x}$ e Alleinoertefob 


\section{Ritarbetter:}

Benerafleutnant a. D. Mar Sdwarte, Exzellend

Oberffleutnant Balter $\mathfrak{A b a m}$

Beneralarzt Dr. Carl Altgelt

Eeneralleutnant Eonftantin bonattrod

Drafor Şans Itenbt

Dberfteutnant Paul \&uguftin

Bebrtreispfarter frang atbert

Beneralleutnant Billiam Bala

Dbert Osuftav von Bartenwerffer

Rorvettentapitån Mar Baftian

Deerfteutnant Siegfries Boelde

Generalinajor $\Re$ u Dolf s. Borries

Cebeimrat Prof. Dr. Paul Elemen

Dberfteutnant ફ̧exmann Eron

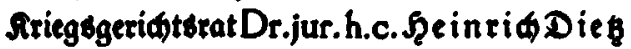

Profeffor Bilbelm Doegen

Ceneralmajor Bilbelm von Dommes

Dberfiteutnant Paul gled

Generalmajor Exid bon flotow

Beneralleutnant $\mathscr{I}$ ugu fi fortmůtler

Generalmajor Şan goft

Oberft Rubolf Gran:

Dbexfteutnant Şand E̊arde

Major J̧ermann Oeyer

Dberfteutnant Eomunb Otaije

Sorbettentapitán Dtto OroD

Liniemidiffoleutnant Yeter greiherr von

Szanbel = \$lazzetti

Stabsapotheler Dr. Mubolf Şanblian

Sonterabmiral Eberbarb Şebbel

Fetbmarjdalleutnant RaF $_{\text {Szoen }}$

Dajor Sart Jૃolfe

Profeffor Şauptmann a. D. Mel d ior

von 5 Z ugo

Éregattenlapitån Emil গ̧uning

Eregattenleutnant $\mathfrak{B}$ il t or $\Im_{g} a l f f g$

von รgaly

Dberf griebrid Immanuel
Dbeeft Iheobor Jodim

Dberftleutnant $\Re$ u o olf $\mathfrak{A}$ ißfling

Dargarete Rlante

Felbmaridalleutnant Theodor Ronto= piecty

Dberfiteutnant Paul srall

General ber Inf. Alfred STauB

Minifterialtat fon on ab $\mathfrak{l} a \mathrm{u}$

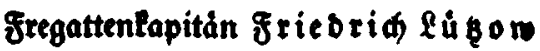

Jelbmar[dalleutnant Jofef $M$ R e ger :

Dberft Balter Nicolai

Dberft Dr. Exid Rigmann

Dberftabobeterinar Dr. Bilbelm Dttto

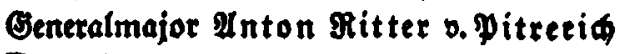

Dr. Elemens Plámann

Dberf Mobert Ritter bon Pobl

Major Erid Prigge

Felbbivifionss und Irmecoberpfarter

Balter Ridter

Dizeabmiral Bernbaro gifing

Şauptmann $\Re$ ubolf Sdmibt

Dberfleutnant $\operatorname{Sarl}$ Sdroeber

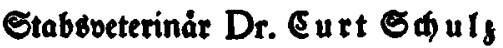

Regierungstat Major a. D. Mubolf Sdu mader

Dr. jur. et rer. pol. $\mathfrak{R} u r t \mathfrak{S} d$ waz

Dberpoftrat f̧ermann Senger

Şauptmann Balter SuBborf

Dberf Beorg Beith

Oberft Stefan oon Belfen

Major Abalbert von $\mathfrak{B a l l}^{2}$ bergg

Dejor friedria $\$$ Bilbelm freibeer oon Billifen

Beneralleutnant $\mathfrak{h} a n$ bon Binterfeclb

Generalmajor Ernft oon $\mathfrak{B r i s b e r g g}$

Generalmajor Ruowig Bur

Beneral ber Infanterie Şans oon 3 woehl 


\title{
Derdeutifase Eandfries
}

Dritter Teil

\author{
$\mathfrak{Z}$ om $\mathscr{W}$ inter $1916 / 1917$

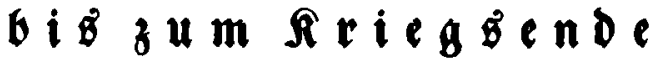

Mit 4 Stizzen auf einer Beilage und 26 Rertifizen

Bearbeitet von

Seneralleutnant Mar Sdwarte, Oberfiteutnant Paul Sted,

Beneralmajor ßubolf ø. Borries, Beneralleutnant Xuguft Fortmüller, Major Jriebrid \$Bithelm Frbr. v. 2 Billifen, Dberfteutnant Sans Barde, Dberft Zheobor Jodim, Seneral ber Jnfanterie Sans v. 3wehl

\section{$\begin{array}{llll}1 & 9 & 2 & 5\end{array}$}

\section{Im gemeinfamen Berlage von:}

Jobann $\mathfrak{A m b r o f i u b ~} \mathfrak{B a r t h}$ in \&eipzig / Deutfiche Berlagbanftalt in Stuttgart

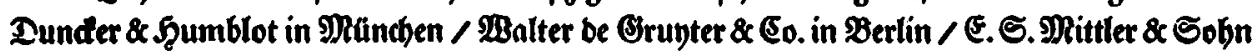
in Berlin / Э. C. B. Mobr (Paul Siebed) in Reibingen / Paul Parey in Berlin. 28. (3. Teubner in Reipzig / Beibmannjhe Bucbjanblung in Berlin Aublieferung für ben Budbanbel bur(b) Jobann Ambrofiub Barth, Reipzig. 
Coppright by Jobann Ambrolius Barth in Reipgtg 1025

Printed in Qenmany 